\title{
La Esperanza en la Experiencia de Sufrimiento Humano. El Psicólogo Humanista y su Ayuda en la Integración del Dolor
}

\section{Hope in the Suffering Experience. Clinical Psychology in Oncological Diseases}

\author{
José Antonio García-Monge \\ Instituto de Interacción y Dinámica Personal
}

\begin{abstract}
Resumen. En el ámbito de la Psicología Humanista y en la corriente de la Psicología Positiva el artículo estudia la ESPERANZA en el dolor y el sufrimiento de los pacientes en psicoterapia en todos los casos de sufrimiento humano y en concreto de los oncológicos. Ponemos la esperanza como tarea básica del Psicólogo clínico en su relación con el enfermo. La psicoterapia como encuentro de dos esperanzas: la del paciente y la del psicólogo. Fundamentamos filosóficamente y antropólogicamente la esperanza y escuchamos sus fuentes para muchos pacientes, en concreto, en la religión (religiones) y en la espiritualidad. Muchas investigaciones aprecian la correlación de las creencias existenciales y de la fe del enfermo con la calidad de vida y la asocian a paz, serenidad, apertura, resiliencia. Disminución de la ansiedad y más fortaleza en los tratamientos. Distinguimos dolor de sufrimiento como elaboración mental del dolor, frecuentemente tortuosa y acrecentadora de ese dolor experimentado. Describimos la tarea del psicólogo clínico humanista para acoger al paciente y, con profundo respeto, a su persona y situación procesar y crear un espacio humano en el que la esperanza diga su palabra.
\end{abstract}

Palabras clave: esperanza, dolor, sufrimiento, psicoterapias humanistas, espiritualidad, amor.

\begin{abstract}
In the field of Humanistic Psychology and current Positive Psychology the article studies HOPE through the pain and suffering in those patients in therapy, in all cases of human suffering and specifically for cancer patients. Hope is the task that we psychologists put forward as a basic task between the clinical psychologist and the patient. Psychotherapy becomes a meeting point between two hopes: the patient's and the psychologist's. We establish the philosophical basis of hope and then we listen to its sources, specifically regarding religion(s) and spirituality. Several researches show the correlation between existential beliefs and the sufferer's faith with quality of life, and it is associated
\end{abstract}

La correspondencia de este artículo puede enviarse a José A. García-Monge. Ex profesor de Psicología Clínica. Universidad Comillas, ICAI, ICADE, Madrid. Profesor del Master de Psicoterapia Individual y de Grupo. Psicoterapeuta Instituto de Interacción y Dinámica Personal. Madrid. Tel 3103240. E-mail jagmonge@yahoo.es 
with peace, serenity, openness and resilience. Decreased anxiety and increased strength for treatments. We distinguish pain from suffering as a mental development of pain, which excruciates and increases the experience of pain. We describe the task of clinical humanistic, psychologists to receive the patient and with a deep respect for him and his situation to create his own human space in which hope is the word.

Key words: hope, pain, suffering, spirituality, love.

\section{La psicoterapia: encuentro de dos esperanzas}

Escribir sobre la esperanza en la experiencia de sufrimiento, como se da en la mayoría de los pacientes con neurosis o crisis existenciales y, en concreto, en el paciente oncológico y sus familiares y amigos, resulta difícil. En muchas ocasiones y circunstancias la única palabra acertada puede ser el silencio. Un silencio empático, vivenciado, tal vez, como impotencia compasiva y comunicada (García-Monge, 2008).

Nuestra tarea (y en psicología clínica no hay tarea sin persona y personas), ante todo hombre, mujer que sufre y en concreto, psico-oncológicamente, pasará por el silencio y la escucha, pero si no acarrea esperanza se quedará corta y no aportará lo que nuestro rol demanda y lo que nuestra persona, desde lo más hondo, puede aportar.

Más allá de las habilidades profesionales, la transmisión de esperanza es una de las más importantes misiones como personas y como profesionales clínicos. La esperanza es un radical humano que no suele aparecer directamente en los libros de psicología, aunque actualmente la Psicología Positiva inaugurada formalmente por Martin Seligman en la conferencia umbral de su periodo presidencial de la American Psychological Association en 1999, integra la esperanza cultivable en la tarea terapéutica. Sin ella, nuestro trabajo relacional en el horizonte existencial queda reducido a estrategias terapéuticas de valor y cierta eficacia desprovista de raíces.

Es verdad que esta "novedad" psicológica de introducir la esperanza, como factor del bienestar, de la calidad de vida, se basa en la reciente fundamentación empírica y una praxis de psicología positiva, pero pertenece a la Psicología Humanista y a la perenne realidad humana. Ya la filosofía moderna (y la antigua, no olvidemos que los primeros psicólogos desde el siglo XIX y primera mitad del XX, provenían de la neurología y sobre todo de la filosofía) sondeaba la esperanza como constitutiva de ese ser finito y limitado que somos abrumado por la soledad, el dolor, el sufrimiento. E. Kant (1787/1998) en su Lógica se encuentra con los límites de la razón en la cuestión: “¿Qué me está permitido esperar?”. Tal vez, como investigaremos en los estudios empíricos, la esperanza en el sentido de la vida o el suprasentido referido desde el sujeto, desde tiempo inmemorial, a Dios (o a los dioses o recientemente a una invisible energía cósmica) constituye la apertura hacia lo suprasensible que permite franquear los límites de nuestra finitud. P. Ricoeur (1969) afirma que los postulados de la razón práctica están ligados a la esperanza, concebida no como "extensión del saber", sino como apertura y que ésta es tanto más necesaria para Kant porque el mal está en la raíz de nuestro querer moral, de tal suerte que "la libertad real no puede brotar más que como esperanza" (Ricoeur, 1969, p. 404). El análisis de la finitud como angustia frente a la nada, como preocupación que surge en el horizonte del tiempo, plantea la cuestión del ser en cuanto ser y, a juicio de Heidegger (1997), así es como Kant lleva el pensamiento filosófico, por encima de Hegel, hacia una ontología existencial. El nihilismo es lo contrario de la esperanza. Genera angustia pero esa angustia puede ser búsqueda de esperanza. Estamos pasando de la metafísica a una antropología filosófica raíz honda del tránsito hacia la psicología.

Además del amor, una de las energías más poderosas que residen en el hombre, aunque su fuente sea más profunda de lo que podemos tocar, es la ESPERANZA.

La esperanza es una virtud, al menos antropológica, un talante, una actitud vital, con resonancia emocio- 
nal, una dimensión de la persona que humanamente es necesaria para que una vida sea digna de tal nombre. El diccionario del uso del castellano María Moliner (1998), define "Esperanza" (abrigar, alimentar, acariciar, concebir) como la "confianza en que ocurra o en conseguir cierta cosa que se desea". Sin placer podemos vivir, aunque frustradamente, sin esperanza, no. Necesitamos la esperanza para que nuestro paso por la existencia no sea receloso, desconfiado, inseguro, doloroso, hostil.

Sin esperanza, que es un bien escaso, no hay vida ni supervivencia, o por lo menos la vida carece de sabor y sentido y la supervivencia sucede sin dignidad. Un médico científico y pensador personalista, Pedro Laín Entralgo (1984), mostró gran interés por la espera y la esperanza. El hombre, escribirá, es constitutivamente un ser que cree, espera y ama. La esperanza, continúa Laín, es un bien absolutamente necesario para las personas, para los grupos, para los pueblos. La esperanza es un anhelo de sentido. La sabiduría popular repite que la esperanza es lo último que se pierde. La esperanza parece ser el ancla de salvación cuando todo se tambalea, cuando padecemos situaciones des-esperadas. Saramago escribe: "La ceguera también es eso, vivir en un mundo en el que se ha acabado la esperanza" (1998, p. 240). La esperanza mira al futuro, desde la experiencia del pasado; está en peligro cuando el ser humano se entrega a la nostalgia, cae en la melancolía o cuando vive un presente "plano" unidimesional. Puede desmesurar el futuro porque es apuesta y riesgo. Pero el riesgo de esperar se ve impulsado por el secreto y hondo corazón de lo humano. La confianza básica que necesita el niño para crecer sanamente está hecha de esperanza en la respuesta de los demás.

En la relación psicólogo/a-paciente se da un encuentro de dos esperanzas. La del psicólogo/a basada en sus conocimientos, su experiencia humana, su motivación y en los recursos del enfermo. La del paciente que, inicialmente puede ser desesperanzada y ¡cuantas veces desesperada! debe ir generándose en la relación terapéutica, en el encuentro humano. Si llegamos a conseguir o, más verdaderamente, si acontece la esperanza en el paciente, habremos creado no una falsa ilusión sino una calidad de vida y sentido en la vida, en muchas ocasiones, más duradero y profundo que la salud misma que tanto y tan motivadamente buscamos.

Escribo en el contexto de la esperanza de sentido de la vida o suprasentido. Frankl explicita que entre las necesidades psicológicas que nos dan sentido a la vida: crear algo, ser útil, amar y ser amado, la tercera, cuando nos encontramos con el sufrimiento, con un destino que parece irrevocable, justamente allí se le puede dar sentido a la vida: "Es el secreto de la incondicionada tendencia al sentido que tiene la vida: que el hombre, precisamente en situaciones límite de su existencia, es llamado a dar fe de aquello que él y sólo él es capaz" (1991, p. 229). Esta afirmación se basa no sólo en una profunda intuición, sino en una laboriosa investigación (1991, p. 230).

Martin Luther King decía: "Si ayudo a una persona a tener esperanza, no habré vivido en vano". Para muchos, el sentido de la vida es, si cabe, más importante que la vida misma.

En este sentido, existe una amplia literatura que confirma la significativa incidencia que tiene el sistema de creencias (que siempre, más o menos sanamente explícita o implícitamente, conlleva esperanza) en la adaptación, calidad de vida e índices de salud de los pacientes y, en concreto de los enfermos oncológicos (Nacional Cancer Institute, 2010). Cuando en estos estudios se habla de religiosidad o de espiritualidad (frecuentemente intercambiables), hay que tener en cuenta que la religión abrazada no es la que más verdades enseña sino la que más esperanza acarrea. Los resultados de un gran número de investigaciones señalan que el poder apoyarse en un sentido de la vida o en un suprasentido espiritual que genere esperanza constituye una forma de afrontamiento para los pacientes más que una forma de evitación o negación, invitándoles al ajuste necesario que supone su enfermedad (Martínez, Méndez y Ballesteros, 2004; Rippentrop, Altmaier y Burns, 2006; Rojas Marcos, 2010). Para la comprensión más honda de esta espiritualidad, religiosidad, nos referimos a, Williams James (1986), uno de los pioneros de la psicología como ciencia, reflexionando sobre la experiencia religiosa.

Por ejemplo, Meyers (2002) en un estudio llevado a cabo con mujeres con cáncer de mama evaluadas tres meses después de su intervención, encontró que, al igual que el locus de control y el apoyo social, la religiosidad influyó de forma significativa en la disminución de la ansiedad experimentada. Midiendo el dolor y la depresión (que es un estado de ánimo frecuentemente desesperanzado), investigadores de un hospital de 
Croacia (Aukst-Margetic, Jakovljevic, Margetic, Biscan y Samija, 2005) encontraron que la religiosidad puede ser un amortiguador de la depresión y un apoyo en el proceso curativo.

Asimismo, los resultados del estudio llevado a cabo por Laubmeier, Zakowski y Bair (2004) desde la perspectiva de la teoría existencial (Frankl, 1963), sugieren que la espiritualidad, particularmente el componente existencial, puede estar asociada con la reducción de síntomas de malestar en pacientes con cáncer, independientemente de la amenaza percibida de la vida.

En la experiencia de dolor vivida en el proceso oncológico o en otros procesos humanos a los que tiene acceso el psicólogo clínico, se da una vivencia sensitiva, cognitiva y emocional desagradable que cada sujeto verifica de manera personal y única. Pronunciar la palabra dolor con empatía, respeto y compasión es relacionarnos con el hecho subjetivamente concreto del paciente.

Existen muchas fuentes de dolor: orgánico, psíquico, social, moral, etc... En el cáncer, como en otras situaciones límite, se puede explicar porqué existe el dolor y paliarlo; al psicólogo humanista y al psico-oncólogo les corresponde adentrarse en cómo se vive o se muere en el dolor y acompañar al sujeto en su tortuoso camino buscando cómo erradicarlo o cómo vivenciarlo con el menor costo personal y con la mayor esperanza posible.

\section{Esperanza: ¿en qué o en quién?}

Hay tantas respuestas como sujetos. La respuesta frecuente es la curación de aquello que le aqueja, además de otras expectativas existenciales. En todo paciente y en el enfermo de cáncer es la salud, su curación, el sentirse útil, no sentirse carga para los demás, que los suyos, a pesar de su enfermedad, salgan adelante, no sufran... Por esta esperanza se afrontan responsabilidades, sesiones de psicoterapia hondas, intervenciones quirúrgicas penosas y largas sesiones de quimioterapia o radio, medicaciones, ingresos hospitalarios, etc...

La esperanza tiene que ver con la necesidad y sobre todo con el deseo. Proyectándolos desde la carencia hacia su realización, el futuro deseado se hace presente en el aquí y ahora esperanzado. Ese futuro puede ser, en muchas ocasiones incierto, tal vez ilusorio, pero el momento actual ha sido esperanzado y esto ya es importante. ¿Nos estamos engañando con la "falsa" esperanza? Puede ser. Pero la esperanza es procesual, evolutiva, como la persona misma.

Aristóteles escribía 300 antes de Cristo que "La esperanza es el sueño del hombre despierto". Despertar a la esperanza es vivir en la realidad del insondable corazón humano, más grande, como señalaba antes, que la misma vida de su organismo biológico. Tal vez una de las tareas del psicólogo es enseñar a desear sanamente o ayudar a quitar las trabas para que esto suceda (Garcia-Monge, 2009). Es verdad que hay "esperanzas" que alienan. No son esperanzas, son sólo ilusorias esperas. Nuestra misión como psicólogos no es engañar ni engañarnos sino respetar y cultivar la esperanza que hace que el dolor, sin dejar de serlo, no se convierta en sufrimiento. Laín Entralgo (1993) muestra su interés por la esperanza en tiempos de crisis.

No se enseña a esperar, pero se puede aprender a tener esperanza. Se puede crear un espacio humano donde haya sitio para la esperanza. Y si logro aprehenderla, existe en mí algo que ni siquiera el fracaso me puede quitar. Puede cambiar el objeto de nuestra esperanza, pero el sujeto que experimenta esperanza está enviando mensajes positivos a su cuerpo, mente, corazón y a su espíritu corporal.

La esperanza no es autosugestión, es ver el arco iris de la vida con todos sus colores. Ampliar la percepción, ensanchar nuestro vocabulario, en el que existen palabras como dolor, muerte y darse cuenta que podemos pronunciar otras palabras que nos hacen explorar todas las dimensiones de nuestro ser, incluido el amor que tanta esperanza genera y tanto dolor, en ocasiones, acarrea. El amor es el "paraíso" perdido y tal vez reencontrado, pero siempre buscado, en esperanza, que nos permite ser-para-alguien y con alguien. No buscaríamos el amor sino hubiésemos sido encontrados por él. El amor hacia sí mismo y hacia los demás está sembrado en la esperanza que nos constituye y nos trasciende. Escribe Frankl (1983, p.110): "El amor constituye la única forma de aprehender 
a otro ser humano en lo más profundo de su personalidad. Nadie puede ser totalmente conocedor de la esencia del otro si no le ama. Por el acto espiritual del amor se es capaz de ver los trazos y rasgos esenciales en la persona amada; y lo que es más, ver también sus potencias; lo que todavía no se ha revelado, lo que ha de mostrarse. Todavía más, mediante su amor, la persona que ama posibilita al amado que manifieste sus potencias. Al hacerle consciente de lo que puede ser y de lo que puede llegar a ser, logra que esas potencias se conviertan en realidad". La esperanza en el amor es más fuerte que la muerte.

Escribía Samuel Johnson en el siglo XVIII: "Es necesario esperar, aunque la esperanza haya de verse siempre frustrada, pues la esperanza misma constituye una dicha, y sus fracasos, por frecuentes que sean, son menos horribles que su extinción".

\section{Dimensiones de la tarea del psicólogo clínico en el asunto de la esperanza}

- Acoger la desesperanza e incluso la desesperación.

- Ayudar en el duelo por la salud perdida.

- Estar atento al mundo cognitivo y emocional del paciente.

- Escuchar sus demandas.

- Respetar sus decisiones.

- Comprender su esperanza, escuchando de dónde le viene.

- Hablarle con esperanza, desde el mundo referencial del paciente.

- Ayudarle a formularla y vivenciarla sanamente.

- Acompañarle en la demora de la gratificación de sus deseos.

- Ahondar junto a él en su esperanza.

- Fortalecer su resiliencia.

Dado que en este artículo no es posible desarrollar todas estas dimensiones me voy a detener en algo que considero importante, el manejo del dolor en todos los pacientes (que padecen) y aterrizar un poco más concretamente, en base a los estudios empíricos, en los pacientes oncológicos.

\section{Dolor y sufrimiento: el manejo del dolor en la Psicología Humanista}

\section{El dolor como dato de la vida}

Aunque la evitación del dolor es antigua como la vida misma, vivimos en una cultura posmoderna en la que como afirmaba Perls (1969) somos "fóbicos al dolor". La trilogía de dioses que motivan conductas humanas, une al dinero y al poder, el culto al cuerpo sano y joven. La experiencia de muerte se oculta para tratar así de olvidar el dolor terminal de la vida.

El dolor, en sus diferentes lenguajes: físico, psíquico, social, moral, espiritual, es un dato de la vida y de las "muertes" agazapadas en nuestra vida. En lugar de aprender a dialogar con él para erradicarlo sanamente o darle un sentido (o conservar, a pesar de él, nuestro sentido), lo anestesiamos pero, tal vez no modificamos la situación vital que nos lo produce. Frecuentemente lo "cambiamos de lugar existencial", lo somatizamos apartándonos, como señala Antonio Guijarro (1979), del sano itinerario que va del síntoma padecido a la vida vivida.

El dolor como dato de la vida podemos:

- Ignorarlo: Alienación, escapismo, autoengaño... hasta que el dolor termina imponiéndose a nuestra negación impotente.

- Evitarlo: Sabemos que está ahí pero evitamos dialogar con él. Tal vez lo sustituimos por otro más llevadero o lo pretendemos compensar de alguna manera. 
- Buscarlo: Masoquismo, victimización, auto-reparación neurótica, culpa como agresión a sí mismo o al entorno.

- Encontrarlo: Escucho lo que me dice, dialogaré con él y la esperanza me ayudará y motivará a fortalecer mi salud personal integral.

\section{Cómo convertimos el dolor en sufrimiento}

Un estímulo, una mutación que genera dolor es frecuentemente, mal o bien, interpretado. Nuestro cerebro, nuestra mente, lo elabora adecuada o inadecuadamente con una actitud positiva o negativa. Esa elaboración mental del dolor que hace el paciente, con toda su biografía a cuestas, puede generar y de hecho genera frecuentemente sufrimiento. La ciencia médica debe tratar de curar la enfermedad que conlleva dolor. La antropología, la psicología, la filosofía, el sentido de la vida, la espiritualidad del ser humano, debe ayudar, con otras herramientas, también a erradicarlo y, en todo caso, a disminuirlo sin convertirlo en sufrimiento que desintegre la persona. El dolor lo encontramos con toda nuestra biografía, con el peso de los aprendizajes, umbrales y expectativas. Coloquialmente intercambiamos las palabras dolor y sufrimiento; sin embargo tienen matices importantes que colorean su vivencia. El dolor es el dato, el sufrimiento es el cómo vivencio, integro en la vida ese dato o me desintegra su existencia (García-Monge, 2009).

\section{Elaboración insana del sufrimiento}

La psicología sabe que no hay enfermedades sino enfermos y cada uno tiene una manera inédita, dependiendo de su personal y social historia, sus recursos, motivaciones, umbrales, de integrar el dolor en la vida doliente o de dejar que el sufrimiento le desintegre. El dolor es el dolor, un dato de nuestra vida biológica, psicológica, social, el sufrimiento es la interpretación personal de ese dolor. Esta interpretación, como hemos señalado puede ser insana:

- Negándolo.

- Instalándolo en nuestra vida, como si nuestro guión existencial sólo se justificara en él.

- Hipostatizándolo: relacionando el dolor con las "fuerzas del Mal" o como erróneas interpretaciones "religiosas”: ¿Por qué a mí?, ¿Qué he hecho para que me toque?, ¿Qué injusticia!. Luis Rojas Marcos (2010) explicando el sufrimiento subraya la importancia no tanto del hecho en sí que nos causa sufrimiento sino de la interpretación personal, subjetiva que, por necesidad de explicar, damos y nos damos como "razón" de ese sufrimiento. La ciencia y la secularidad, así como una correcta interpretación de la religión, han exorcizado a los "dioses del mal" y resituado el dolor en el azar, la finitud, la fragilidad o la perversa voluntad de los hombres.

El dolor, sobre todo cuando es profundo y duele mucho, es una de esas experiencias humanas que son difíciles de integrar (que no supone sólo comprender ni justificar, ni mucho menos exaltar). Pide, ante todo respeto, silencio y empatía. El dolor produce un vértigo de soledad, posiblemente algo paliado por el apoyo social, que experimenta "el hombre doliente", la soledad del "corredor de fondo". El gran neurólogo, creador de la logoterapia, Viktor E. Frankl, llega a afirmar que el dolor es una oportunidad de madurar, de encontrar sentido, esperanza, de crecer si se sabe manejar auténticamente (1987, pp. 249-265). Y con la voluntad de sentido puede llegar muy profundamente al corazón y espíritu del hombre abierto a un suprasentido. Frankl hace, con respeto y claridad, el elogio de la dignidad del hombre doliente y nosotros nos sumamos a él. 


\section{Sufrimiento e Integración personal}

Señalaré algunas actitudes que, convertidas en conductas operativas, pueden ayudar al psicólogo clínico humanista a crear el espacio terapéutico en el que el "hombre doliente" pueda crecer personalmente.

- Escuchar cómo vive el dolor el paciente y ayudarle a relacionarse con realismo con este dato de su vida redimensionándolo. He escrito "con realismo" pero ese realismo en la persona humana es mayor que un diagnóstico. No se trata de esperar milagros sino de reconocerse no sólo en lo que se sufre sino en lo que se es.

- Ayudar a no confundir el Mal con el dolor, tal vez por secuelas que nos quedan de nuestro animismo infantil.

- Inducir respetuosamente a no autoagredirse.

- Reconocer la parcela de responsabilidad, si la hay, y ampliar la mirada a la dimensión salud que siempre, en algún rincón de la persona, existe. No con falsos consuelos o racionalizaciones, sino con mirada amplia y profunda que se puede ir dando en el desarrollo del vínculo terapéutico. Es verdad que el paciente es aquí y ahora este dolor, pero también es cierto que es más que este dolor.

- Encontrar, como escribía Viktor Frankl (1987, pp. 249-265), mi sentido en el dolor. El sin sentido es causa profunda de sufrimiento. El dolor me puede arrebatar mucha vida pero no la voluntad de sentido, el último reducto de la libertad de nuestra desnuda existencia. El dolor puede ser de parto que dará a luz vida o luz a la vida doliente. Aquí el amor tiene una palabra importante que decir, aunque también puede ser causa de especial dolor. Recordando las tres dimensiones del amor tal como Rubin y otros psicólogos han estudiado (Sternberg, 1989): intimidad, pasión y compromiso, vemos que pueden generar mucha esperanza y ser un motivo hondo para la vida, sea cual sea, su circunstancia. Así lo muestra la experiencia. El amor es una apuesta por la vida y, en ocasiones al menos, es más fuerte que la vida. Es cierto que el amor nos hace vulnerables acogiendo en el corazón el sufrimiento de otras personas que nos tocan, pero el amor a una(s) persona(s), a una causa, a una tarea, pueden redimensionar nuestro dolor. Dolor es una penúltima palabra, amor es la última y definitiva. Aprender a dejarse amar y amar en la experiencia dolorosa da testimonio de valores que constituyen el patrimonio profundo del ser humano (Fromm,1985). Por parte del psicólogo clínico esta experiencia de hombre, mujer psicoterapeuta humanista no será ni fiable ni profunda sino se basa en el amor lúcido al paciente.

\section{Recursos gestálticos en la elaboración del sufrimiento}

Estos recursos tienen sus raíces en lo que llevamos escrito anteriormente. Son como la punta de un iceberg que se basa en su profundidad.

Ayudar ("acompañar cuidando" es la etimología griega de la palabra terapia) a vivir el presente, el aquí y ahora. La anticipación catastrófica del futuro o la evasión del pasado evitan ponerme en contacto conscientemente con mi presente, aunque sea doloroso, pues ahí debe nacer la aceptación y sembrarse la esperanza verdadera.

Tener en cuenta que el pensamiento omnipotente infantil puede aumentar el sufrimiento. Enviar mensajes, a través de un pensamiento positivo, que amplían la consciencia y fortalecen la resiliencia. Frecuentemente la visualización positiva ayuda en esta tarea, así como las técnicas de relajación e incluso la práctica de la meditación por los cambios que genera en el cerebro y el sistema endocrino. Ayudar a atajar maniobras de evitación, ampliando el realismo de la verdad en tanto en cuanto el paciente se puede ir abriendo a ella.

Preguntas que pueden ampliar la awareness y dar palabra a la responsabilidad, como: "¿Qué estoy evitando con el sufrimiento?” " “Qué 'beneficio’ (secundario e insano) saco con el sufrimiento?” pueden 
ayudar en sus balbuceos de respuesta progresivamente consciente. Puede que esté evitando responsabilizarme de mí mismo o, aunque me parezca obvio que no saco ningún beneficio, tal vez alguno, más o menos consciente, me esté manipulando o desde él esté manipulando al entorno. "¿Qué precio pago con el sufrimiento?": ser centro, autocastigarme, agredir, tener ocasión de rebelarme o manifestar mi sin sentido.

Es muy importante ahondar en el: “¡A quién o a qué he dado poder de hacerme sufrir?”. Damos, frecuentemente, poder excesivo a circunstancias, personas, realidades, que nos hacen sufrir ciertamente porque les hemos dado poder, tal vez desmesurado, de "quemar" nuestra persona. Esto acontece diariamente. Mis emociones se despiertan dolorosas porque "he decidido" llamar fracaso a este dolor o ser esclavo de esta carencia o desmesurar la importancia de esa persona. Aparentemente no damos poder de hacernos sufrir pero, de hecho, lo tienen muchas personas o circunstancias que pueblan nuestro espacio real o afectivo o que no encajan con nuestro autoconcepto y sus deseos y necesidades. Si la lista de "poderes otorgados" es muy grande, he multiplicado las identificaciones de mi yo y me he hecho más vulnerable. Como el rico que tiene más cosas que pueden ser robadas. El paso del ser al tener, como señala Erich Fromm, es fuente de sufrimiento. Sin tener algo no es fácil ser, se sufre, duele; teniendo mucho es más difícil ser, a la larga y, en alguna dimensión de la vida, se sufrirá más, aunque parezca lo contrario.

¿Qué es lo peor que puede ocurrir en esta situación? Una pregunta que el psicólogo humanista puede dejar abierta a los temores del paciente. Puede ocurrir que su respuesta más consciente y profunda, disminuya los miedos y compruebe que, en ocasiones, desmesuramos las expectativas catastróficas, fantasías aterradoras. Hacerme consciente de los temores no debe impedir conocer mis recursos.

¿Con qué cambio superaría o, al menos integraría mejor, mi sufrimiento? No solemos concebir el final del sufrimiento sin un cambio y, casi siempre, ese cambio tiene que ver con el afuera, con la realidad en torno más que con la propia actitud. Frecuentemente es cierto, pero, en muchas ocasiones, el paciente, ayudado por su psicoterapeuta puede preguntarse: ¿Qué tengo yo que cambiar?, ¿Elijo cambiarlo? Se trata de llevar, en la medida de lo posible, el locus de control hacia la libertad del paciente, en la medida de sus recursos, más grandes de lo que a primera vista puede parecernos. ¿Qué hago para no cambiar? Resistencias, desconfianza, desesperanza, baja autoestima. Viktor Frankl (1983) citando a Nietzsche escribe: "Hay mucha sabiduría en Nietzsche cuando dice: 'Quien tiene un porqué para vivir puede soportar casi cualquier cómo'”. En otro lugar he escrito sobre el porqué para vivir, para el crecimiento personal (García-Monge, 2002). Es cierto, como he señalado anteriormente, que el hombre es un ser finito, limitado (el dolor y el sufrimiento tiene que ver con esa realidad) pero, como señala Frankl (1983, p. 124): "Cierto, un ser humano es un ser finito, y su libertad está restringida. No se trata de liberarse de las condiciones, hablamos de la libertad de tomar una postura ante esas condiciones".

Optar por vivir, no por durar. La vida es más grande que el organismo vivo. El gran enemigo de esta opción no es solamente el sufrimiento sino el vacío existencial. En este vacío está ausente la esperanza, el amor, el sentido. Va más allá del dolor. Se trata de un sufrimiento mental en el que el corazón está inapetente. Se ha muerto el deseo. El ser humano entonces es algo, una cosa que funciona y funciona mal. No se trata de "llenar" el vacío con vaciedades sino de descubrir mi propio ser con un sentido o un suprasentido que da razón de mi esperanza o da la razón a mi esperanza y otorga credibilidad a la confianza en la honda intuición del corazón humano. No dejemos que la nube nos haga pensar que el sol no existe.

El psicólogo humanista debe sondear y cuidar la imagen de la felicidad que tiene el paciente. Tal vez es una imagen culturalmente vendida o prestada que está introyectada pero no es una imagen sólida y personal sanamente internalizada. Cambiar esa imagen implica cambiar expectativas y ahorra sufrir frustraciones dolorosas.

Estoy firmemente convencido de que el dolor cabe en nuestra vida, pero la totalidad de nuestra vida no cabe en nuestro dolor. Tal como he señalado en otro lugar (García-Monge, 1996) “"somos más grandes que la enfermedad”. El psicólogo clínico debe ser consciente de la enfermedad pero debe mirar lo que de salud existe en 
el paciente. Debe enseñar a dialogar con la salud personal que, tal vez, esté escondida entre los escombros de la enfermedad.

Escuchar el mensaje del dolor y actualizar todos nuestros recursos para erradicarlo o disminuirlo sin permitir que "queme" nuestra persona.

Séneca, filósofo romano-español (siglo I) escribía: "Cuando no se puede sufrir algo, lo mejor es saberlo sufrir" o "Lo importante no es lo que sufres sino cómo lo sufres".

El sufrimiento depende no tanto de lo que se padece, cuanto de cómo se padece y de nuestra imaginación que aumenta nuestros males o puede disminuirlos con una intuición apoyada en una fe y en un sistema de creencias sanas, válidas para el sujeto y, mucho más si las comparte con una comunidad o grupo.

El mejor servicio que podemos prestar a los que sufren no es quitarles la carga (si podemos estaría muy bien pero frecuentemente será difícil o imposible) sino ayudarles a encontrar la energía necesaria para sobrellevarla.

\section{A modo de conclusión}

Aceptación es una palabra clave en toda la vida, en todo humano dolor y también en psico-oncología. Aceptar la enfermedad, no sin lucha y rebeldía, en todo itinerario humano, es difícil. Aceptar que, con el dolor y, en concreto con el cáncer, podemos seguir siendo aceptados y, profundamente nos aceptamos (eso tiene que ver con amados) nos revela algo o, tal vez, Alguien, que habla del objeto de nuestra más radical esperanza.

La desesperanza se basa en lo que sabemos, que es nada o muy poco, y la esperanza sobre lo que ignoramos que es todo o casi todo. Nunca la humanidad ha estado tan informada. La medicina diagnostica con aproximada exactitud. Si miramos nuestra historia cotidiana la información no nos aporta demasiada esperanza por causas que no queremos interesadamente cambiar o no podemos modificar. Pero la esperanza es patrimonio de la humanidad. No pertenece a los "saberes" sino a la sabiduría. Aprendemos conocimientos, está bien, pero sin esperanza esos conocimientos nos remiten a una dolorosa impotencia. Ante todo paciente y en concreto el oncológico, sabemos lo que le afecta en deterioro de su salud, pero él nos puede enseñar que es mucho más que un organismo enfermo, que las células cancerígenas no hacen metástasis en su espíritu. Esto puede parecer muy poco o, incluso ilusorio, pero da un rostro profundamente humano a la vida y a la muerte. Todos hemos conocido enfermos así: ¿he aprendido algo que me puede enseñar a vivir?. Son pobres, en salud, que nos enriquecen. Nosotros somos ricos profundamente pobres. Pensamos que somos lo que tenemos (sanos si tenemos salud) pero no somos lo que, en realidad, está seminalmente sembrado en nuestro ser. Creer en esto se llama esperanza. El que siembra deja morir el grano, el que cosecha lo recibe multiplicado. Al sembrar sólo nos queda la esperanza. El psicólogo/a clínico siembra, día a día, con trabajo, vida. Si esa vida no la transita el dinamismo de la esperanza daremos tiempo a la vida, no vida al tiempo, a la persona temporal.

El sufrimiento encadena, nos hace egocéntricos. En algunos casos nos aferra a la cama, o a nuestras neurosis, nos hace depender del tratamiento; limita nuestra autonomía. Esa realidad limitante se puede vivir mejor o peor. Mejorándonos como personas o empeorándonos. La esperanza es una dimensión, a veces la única, de libertad. No pensamos en una ausencia de condicionantes (obvios en la enfermedad), sino en la capacidad de dar sentido a pesar y en esos condicionantes. Somos libres para esperar, para experimentar esperanza. Cualquier crisis existencial y el cáncer pueden deteriorar partes de nuestra mente o de nuestro organismo, pueden amenazar la esperanza que existe en nosotros como don y tarea, pero no pueden acabar con ella.

Es verdad que el acontecimiento de la muerte está inexorablemente sembrado en el itinerario humano. Se puede experimentar, y el existencialismo lo hizo, que somos un ser-para-la-muerte, pero también se puede esperar (no hablo sólo del más allá) que la mujer, el hombre es un ser-para-la-vida. Y vivenciar esto tan hondamente, como lo han hecho muchos seres humanos, nos dice que la Vida es más grande que nuestras muertes e inclu- 
so que nuestra muerte. Desde el amor que-se-da-a-los- demás, pensemos en el altruismo verdadero tal como nos lo describe la Psicología Social (Yela, 2002). Podemos justamente temer la muerte, aunque este temor puede estar modificado por la mente, que es la avería definitiva de nuestro organismo biológico, con muchas consecuencias existenciales y sociales, pero, en ocasiones, experienciamos tan fuertemente la fuerza de la vida, sobre todo cuando va energetizada por el Amor, que la real amenaza de la muerte se debilita ante la consistencia de una Vida dinamizada por la esperanza y realizada por el amor.

Esperar es, a veces, desear lo imposible. Pero, ¿quién nos prohíbe desear lo humanamente imposible? Responderéis que el sentido común, el realismo. Es verdad, pero no toda la verdad. Todo lo científico es real, pero no todo lo real es científico. La esperanza que acarrea amor es el corazón de la realidad.

\section{Referencias}

Aukst-Margetic, B., Jakovljevic, M, Margetic, B., Biscan, M. y Samija, M. (2005). Religiosity, depression and pain in patients with breast cancer. General Hospital Psychiatry, 27(4), pp. 250-255.

García-Monge, J.A. (1996). El manejo del dolor en psicoterapia. En C. Alemany y V. García (Eds.), El cuerpo vivenciado y analizado. Bilbao: Desclèe de Brouwer.

García-Monge, J.A. (2008). Perspectiva psicológica de la compasión. En M. García-Baró y A. Villar (Eds.): Pensar la compasión. Madrid: Universidad Pontificia Comillas.

García-Monge, J.A. (2009). Treinta palabras para la madurez. Bilbao: Desclèe de Brouwer.

García-Monge, J.A. (2002). El camino hacia el porqué. En C. Alemany (Ed.): Relatos para el crecimiento personal. Bilbao. Desclée de Brouwer.

Frankl, V. (1991). La voluntad de sentido. Barcelona. Herder.

Frankl, V. (1987). El hombre doliente. Fundamentos antropológicos de la psicoterapia. Barcelona: Herder.

Frankl, V. (1983). El hombre en busca de sentido. Barcelona: Herder.

Fromm, E. (1985). El arte de amar. Barcelona: Paidos.

Moliner, M. (1998). Diccionario del uso del español. Madrid: Gredos.

Nacional Cancer Institute (2010). La espiritualidad en el tratamiento del cáncer. Consultado online, febrero de 2010. http://www.cancer.gov/espanol/pdq/cuidados-medicos-apoyo/espiritualidad/ healthprofessional

Heidegger, M. (1997). Caminos del bosque. Madrid: Alianza.

James, W. (1986). Las variedades de la experiencia religiosa. Barcelona: Península.

Kant, E. (1998). Crítica de la razón pura. Madrid: Alfaguara (Edición original publicada en 1787).

Laín Entralgo, P. (1984). La espera y la esperanza: historia y teoría del esperar humano. Madrid: Alianza.

Laín Entralgo (1993). Esperanza en tiempo de crisis: Unamuno, Ortega, Jaspers Bloch, Marañón, Heidegger, Zubiri, Sartre, Motmann. Barcelona: Galaxia Gutemberg .

Laubmeier. K.K., Zakowski, S.G. y Bair, J.P. (2004). The role of spirituality in the psychological adjustment to cancer: A test of the transactional modelo of stress and doping. International Journal of Behavioral Medicine, 11(1), pp. 48-55.

Martínez, M.R., Méndez, C. y Ballesteros, P. (2004). Características espirituales y religiosas de pacientes con cáncer que asisten al Centro Javeriano de oncología. Universitas Psychologica, 3 (2), pp.231-246.

Meyers, K. R. (2002). Religiosity and health locus of control as predictors of depression and anxiety in woman with breasts cancer. Dissertation Abstracts International. Seccion B: The Sciences and Engineering. Vol. 63 (6-B), pp. 3016.

Perls, F. (1969). Gestalt therapy verbatim. New York: Batan Books

Guijarro, A. (1979). Integracion emocional y Psicología Humanista. Madrid: Marova.

Ricoeur, P. (1969). Le conflit des interprétations. Paris: Seuil.

Rippentrop, A.E., Altmaier, E.M., y Burns, C.P. (2006).The Relationship of Religiosity and Spirituality to 
Quality of Life Among Cancer Patients. Journal of Clinical Psychology in Medical Settings, 13 (1), pp. 3137.

Rojas Marcos, L. (2010). Superar la adversidad. El poder de la resiliencia. Barcelona: Espasa.

Sternberg, R.J. (1989). El triángulo del amor. Intimidad, pasión y compromiso. Barcelona: Paidós.

Saramago, J. (1998), Ensayo sobre la ceguera. Madrid: Alfaguara.

Yela, C. (2002). El amor desde la Psicología Social. Ni tan libres, ni tan racionales. Madrid: Pirámi-de.

Manuscrito recibido: 17/02/2010

Revisión recibida: 01/03/2010

Manuscrito aceptado: 04/03/2010 